\title{
Extending drug ethno-epidemiology using agent-based modelling
}

\author{
David Moore', Anne Dray², Rachael Green', Susan L. Hudson³, Rebecca Jenkinson ${ }^{4}$, \\ Christine Siokou', Pascal Perez², Gabriele Bammer ${ }^{5}$, Lisa Maher ${ }^{6}$ \& Paul Dietze ${ }^{4}$ \\ National Drug Research Institute, Curtin University of Technology, Australia,' Research School of Pacific and Asian Studies, The Australian National University, \\ Australia, ${ }^{2}$ School of Public Health and Community Medicine, University of New South Wales, Australia, ${ }^{3}$ Monash Institute of Health Services Research, Monash \\ University, Australia, ${ }^{4}$ National Centre for Epidemiology and Population Health, ANU College of Medicine, Biology and Environment, The Australian National \\ University, Australia $^{5}$ and National Centre in HIV Epidemiology and Clinical Research, University of New South Wales, Australia ${ }^{6}$
}

\section{ABSTRACT}

Aims To show how the inclusion of agent-based modelling improved the integration of ethno-epidemiological data in a study of psychostimulant use and related harms among young Australians. Methods Agent-based modelling, ethnographic fieldwork, in-depth interviews and epidemiological surveys. Setting Melbourne, Perth and Sydney, Australia. Participants Club drug users in Melbourne, recreational drug users in Perth and street-based injecting drug users in Sydney. Participants were aged 18-30 years and reported monthly or more frequent psychostimulant use. Findings Agent-based modelling provided a specific focus for structured discussion about integrating ethnographic and epidemiological methods and data. The modelling process was underpinned by collective and incremental design principles, and produced 'SimAmph', a data-driven model of social and environmental agents and the relationships between them. Using SimAmph, we were able to test the probable impact of ecstasy pill-testing on the prevalence of harms - a potentially important tool for policy development. The study also navigated a range of challenges, including the need to manage epistemological differences, changes in the collective design process and modelling focus, the differences between injecting and non-injecting samples and concerns over the dissemination of modelling outcomes. Conclusions Agent-based modelling was used to integrate ethno-epidemiological data on psychostimulant use, and to test the probable impact of a specific intervention on the prevalence of drug-related harms. It also established a framework for collaboration between research disciplines that emphasizes the synthesis of diverse data types in order to generate new knowledge relevant to the reduction of drug-related harms.

Keywords Agent-based modelling, Australia, drug policy, ethno-epidemiology, psychostimulants.

Correspondence to: David Moore, National Drug Research Institute, Melbourne Office, 7/19-35 Gertrude Street, Fitzroy, VIC 3065, Australia. E-mail: d.moore@curtin.edu.au

Submitted 10 December 2008; initial review completed 17 April 2009; final version accepted 11 June 2009

\section{INTRODUCTION}

During the 1980s and 1990s, there was increasing recognition within drug research of the explanatory limitations of quantitative approaches and the limited generalizability of ethnographic studies [1]. One consequence was the development of drug ethno-epidemiology [2]. This approach emphasizes 'cross-methodological and analytical dialogue' across multi-disciplinary research teams [3]: it combines ethnography's focus on local contexts with epidemiology's focus on populations, and allows for iterative interplay between the different levels. Ethnographic research informs the design and interpre- tation of epidemiological studies and, in turn, epidemiological research guides ethnographic investigations of key topics and tests ethnographic findings in larger samples (e.g. [3-5]). In this way, some of the limitations of the two forms of data collection are minimized (e.g. ethnography's specificity and the limited depth of quantitative instruments), some of their advantages are reinforced (e.g. ethnography's richness and the use of large samples in epidemiology) and their interactive potential is maximized.

Our ethno-epidemiological work on psychostimulant use and related harms among young Australians extended this dialogic tradition by including a formal 
integrative component-agent-based modelling-to overcome a key barrier to the development of mixed methods research, the genuine integration of data [6]. As Bammer [7] has argued, developing and using models can be a valuable process for achieving integration of different types of knowledge. We decided on agent-based modelling because of its ability to provide insights into complex systems [8-13], and used it to build a simulation platform that could integrate our ethno-epidemiological data and test various scenarios, such as the probable impact of specific interventions on the nature and prevalence of psychostimulant-related harms. In this paper, we describe how we used agent-based modelling to integrate ethno-epidemiological data, provide an example of the insights made possible by the modelling and discuss some of the challenges raised by such work.

\section{THE RESEARCH ISSUE}

Our research focused on psychostimulant use and related harms. Between 1991 and 2004, the period preceding our research, national surveillance systems recorded increases in the 'recent use' of psychostimulants (i.e. use in the last 12 months), with more than $10 \%$ of $20-29$ year-olds reporting recent use of methamphetamine or ecstasy in 2004 [14]. This pattern of relatively high levels of use among young Australians echoes international trends, with psychostimulants ranked consistently among the most widely used drugs in the Asian region [15,16]. The harms associated with psychostimulant use include dependence, increased injecting risk practices, violence, physical health problems (e.g. cardiac arrhythmia, renal problems and seizures) and mental health problems (e.g. anxiety, depression and psychosis) [5,17-20].

In Australia, the rising prevalence of psychostimulant use (particularly the use of crystal methamphetamine or 'Ice') and related harms has been the subject of political concern (e.g. [21,22]). This was fuelled by data on trends in drug use from Australian surveillance systems. However, these systems are unable to undertake the enriching follow-up studies required to understand more clearly the social and cultural factors that underlie changing trends in use and harms [23]. Ethnographic and other qualitative approaches that seek to provide such information (e.g. [24,25]) focus on specific geographical or social settings, which means that the findings cannot be generalized to larger populations. The situation in Australia was therefore ripe for an ethnoepidemiological study of psychostimulant use and related harm, the first to be conducted in this country.

\section{ETHNO-EPIDEMIOLOGICAL METHODS}

Quantitative and qualitative data collection focused on psychostimulant use among club drug users in Mel- bourne; recreational drug users in Perth (i.e. those using psychostimulants in private as well as in public settings such as clubs and bars); and street-based injecting drug users in Sydney. Participants were aged 18-30 years and had used psychostimulants at least monthly for the last 6 months. These populations and locations were chosen for study because of their high prevalence of psychostimulant use. The three research sites provided a broad sampling of psychostimulant contexts in order to gain insights into a variety of geographical, demographic and cultural ecologies [5] that shape psychostimulant-related harms.

The ethnographic component (conducted by C.S. in Melbourne, R.G. in Perth and S.H. in Sydney) ran from September 2005 to July 2007 [26,27]. It involved participant observation with approximately 250 young psychostimulant users in natural settings such as streets, clubs, music festivals and private parties. Sixty-four in-depth interviews were also conducted across the three fieldsites. The epidemiological component (conducted by R.J.) consisted of two surveys conducted concurrently with the ethnographic fieldwork. The first survey $(n=363$ across all sites) was conducted in February-May 2006 and used a shortened version of existing surveillance instruments to canvass patterns of psychostimulant use and related harms [28]. The second survey $(n=248$ in Perth and Melbourne) was conducted in September 2007-March 2008 and used a version of survey 1 that was modified on the basis of the insights obtained through ethnographic fieldwork and preliminary analysis of data collected in survey 1 .

The ways in which the ethnography and epidemiology informed one another in our project echoed the crossmethodological dialogues described in previous ethnoepidemiological work. For example, on the basis of the ethnographic findings, survey 2 added a new section designed to elicit detailed information about alcohol use and its relationship to patterns of psychostimulant and other drug consumption; preliminary analysis of survey 1 revealed a marked discrepancy between the prevalence of diverted dexamphetamine use in the Perth (74\%) and Melbourne samples (10\%), which reinforced an emerging ethnographic focus on such use in Perth [26]. However, we also included agent-based modelling as a core component of our study, with the modelling evolving in conjunction with the epidemiological and ethnographic arms.

\section{DEVELOPING AN AGENT-BASED MODEL}

The modellers involved in the project (A.D. and P.P.) concentrated on the development of 'SimAmph', a datadriven model of social and environmental 'agents' (i.e. young people, psychostimulants and settings for drug 
use) and the relationships between them [29,30]. SimAmph offered the opportunity to combine ethnographic and epidemiological data, micro- and macrolevel information, and the individual, social, temporal and spatial dynamics of psychostimulant use [31,32]. Once the agents and relationships had been established, we could run simulations in which, by varying agent characteristics or the relationships between them, we could test various scenarios. For example, we could assess the potential impact of an intervention on the nature and prevalence of psychostimulant-related harms.

The process of developing SimAmph was guided by two principles. First, collective design [33] involved the input of data and concepts produced by the ethnographers and epidemiologists and iterative questioning by the modellers of these data and concepts, which allowed the research team to create a common ontology over time. Secondly, incremental design [34] led to the development of an initial agent-based model which was then modified through the collective design process, thus allowing for partial verification at each stage of the process. Because the research team was located physically in four cities (Perth, Melbourne, Canberra and Sydney), with data collection occurring simultaneously in three research sites, adhering to these design principles required regular teleconferences, e-mail discussions and face-to-face meetings between team members.

The modellers began by attempting to produce preliminary models for each field-site prior to integrating them into an overarching generic model. These models included the social and environmental agents relevant to psychostimulant use in each city and the rules governing their interaction. These were drawn from ongoing ethnographic and epidemiological data collection and elaborated and refined further during team discussions. The ethnographers sent summaries of their fieldwork data to the modellers approximately every 3 months, while the epidemiological data supplied the patterns and prevalence of psychostimulant use and related harms. In order to manage the potentially sensitive issue of data ownership, we agreed that primary data sets would remain the intellectual property of the corresponding researchers and their institutions.

Attempting to develop the city-based models led to the identification of several gaps in knowledge (city-specific or relating to all sites) which were addressed in ongoing ethnographic fieldwork and which informed the design of survey 2. For example, how did the consumption of psychostimulants differ between private and club settings? How did young psychostimulant users conceptualize the balance between the benefits of their drug use and any associated harms? To what extent did law enforcement activities shape local patterns of drug use?
However, integrating the three case studies into a generic model proved impossible for two reasons. First, building models of psychostimulant use for each city was overly ambitious, given the limited focus of our ethnoepidemiological work in specific social networks and convenience samples of young people. We did not have the information required to develop city-based models in a meaningful way or the necessary resources to collect such information (e.g. information on psychostimulant use among other populations in the three cities). Secondly, even if we restricted our focus to young people, the marked differences between the injecting and noninjecting samples identified in the ethnographic data and survey 1 made integration difficult. The social contexts of use and harms associated with street-based injecting of psychostimulants (Sydney) were fundamentally different from those associated with the recreational use of psychostimulants in private homes (Perth) or clubs (Melbourne).

The commonalities emerging across the Melbourne and Perth sites suggested an alternative strategy: an exclusive focus on these case studies in both the modelling and survey 2. Instead of a city-based model, we developed one based on psychostimulant 'trajectories'. We chose trajectories because the ethnographic and epidemiological data suggested that young people moved through distinct stages of involvement in psychostimulant use.

The next step was to create a simulation model using the Cormas platform [35]. Preliminary simulations were run to test the conceptual coherence of the model and to ensure that it captured adequately important aspects of the social contexts of psychostimulant use. On several occasions, this process led to further identification of knowledge gaps as well as modification of SimAmph's structure. This iterative process led to the collective acceptance of a final version of SimAmph.

As noted by one team member, the modelling process evolved as a 'tool for managing the conversation', an ongoing forum for structuring dialogue between the ethnographic and epidemiological components. It provided opportunities for team members to develop collective interpretations of data, to discuss the possible integration of the Sydney sample and decide eventually on the Perth/ Melbourne modelling focus, and to think systematically about the relationship between their data and the emerging model. This model is described next.

\section{SIMAMPH}

SimAmph focuses on stages of involvement in weekend psychostimulant use and their relationship to harms. It includes human and pharmacological agents and their characteristics: young psychostimulant users (e.g. their 
socio-demographics, health status, motivations for involvement and the nature of their peer networks); and the drugs they consume routinely (e.g. drug typesalcohol, cannabis, ecstasy, methamphetamine powder and crystal methamphetamine-and associated patterns of use). SimAmph also includes spatial agents and their characteristics: that is, the settings of drug use (i.e. private and public settings; and the degree to which drug use is accepted in these settings). Finally, it includes attention to the ways in which drugs are represented in public discourse (e.g. through media coverage).

Each human agent falls into one of five stages of psychostimulant use:

- 'novice' (consumes alcohol and cannabis regularly but is yet to experiment with psychostimulants or has used them on a few occasions);

- 'occasional' (consumes psychostimulants monthly or less);

- 'regular' (consumes psychostimulants weekly to monthly);

- 'hardcore' (engages in frequent weekend 'benders' lasting 1-3 days); and

- 'marginal' (uses psychostimulants daily).

Because human agents move between stages, SimAmph also includes the 'stage dynamics' or rules of transition-in other words, the reasons young people move between the different stages (e.g. increasing psychostimulant use because of the associated pleasures, decreasing use because of escalating health-related harms). Drawing on our ethnographic data, we developed two such rules. First, young people increase their involvement in psychostimulant use through a "peer influence' parameter which, in SimAmph, is a function of the drug-using patterns of friends in a particular stage, the drug-related norms of the venues they typically visit and the media positioning of psychostimulants. Secondly, young people decrease their involvement in psychostimulant use through a 'health experience' parameter, which is a function of the mental and physical health of friends and self, as these are operationalized in the model.

Finally, we included the various harms associated with psychostimulant use (i.e. physical, psychological, social and acute) along with their frequency (using prevalence data from surveys 1 and 2). We calculated the probability of experiencing specific harms and their probable impact over the short term (defined as three or more occasions of psychostimulant use) and long term (defined as 80 or more occasions of psychostimulant use).

SimAmph thus incorporated a range of parameters: the size and socio-demographic characteristics of the population to be modelled, the stages of involvement in psychostimulant use (including patterns of drug use associated with each stage), the social and health levers that increased or decreased involvement in these stages, the influence of different settings on levels of use and the probabilities of experiencing various drug-related harms. The model allows for transparency and flexibility of use, and most of the parameter values can be accessed and updated as new data emerge.

In order to validate the model, we ran a base scenario that began with a population of 200 agents (located initially in a pre-stage 'pool') and simulated their involvement in psychostimulant use over 3 years. Drawing on ethnographic data, we assumed three distinct periods of use: (i) a 'honeymoon' period of initial psychostimulant use where a human agent's peer influence value increases rapidly while the effects on health are yet to develop; (ii) a 'transition' period where the increasing experience of adverse health consequences leads to a re-assessment and decrease in use; and (iii) a 'normalization' period, in which stabilizing values of peer influence and health experience establish fixed or cyclic patterns over time. At the end of the 3 years, the approximate percentages of young psychostimulant users in each of the five stages of involvement were reasonable proxies of those derived from the epidemiological surveys. This suggested that the model was relatively robust and ecologically valid [36,37].

Building the agent-based model allowed us to test some 'what-if' situations. We ran simulations to model the potential impact of various interventions by making empirically based assumptions about how changes in agent characteristics or relationships between them would play out. We describe one of these simulationsfocusing on ecstasy pill-testing-below. We provide only one simulation here to give a flavour of what is possible using the modelling. Further simulations - on the probable impact of law enforcement and drug educationwill be published elsewhere.

\section{MODELLING THE POTENTIAL IMPACT OF ECSTASY PILL-TESTING}

We chose to model the impact of ecstasy pill-testing for several reasons. Adulterated pills are a regular feature of the ecstasy market [38], and 'overdoses' occur periodically as a result of the consumption of 'ecstasy' pills that contain drugs such as paramethoxyamphetamine (PMA). Existing evidence suggests that pill-testing is seen by young psychostimulant users as an 'effective and feasible way of avoiding "bad” pills' ([38], p. 62). Such testing is one (albeit contested) option in the Australian drug policy landscape as well as being a feature of harm reduction initiatives in other countries such as the Netherlands [39].

The question we asked was: if the ecstasy market is 'invaded' by adulterated pills and the market penetration 
of these pills varies between 10 and $50 \%$, what percentage of young psychostimulant users would need access to pill-testing in order to ensure that the prevalence of a major medical condition (defined as an overdose or other situation requiring medical intervention) remained at 'usual' levels (i.e. $<5 \%$ according to data from survey 2 )?

Our hypothetical situation was that the market invasion by adulterated pills occurs at 52 time-steps (implemented as weeks in SimAmph) into the simulation and that this situation lasts for 10 weeks. We assumed that if young psychostimulant users consumed these adulterated pills, they would have a $100 \%$ chance of developing a major medical condition. On the basis of our research findings and those reported in previous studies $[38,40,41]$, we also assumed that: (i) young psychostimulant users would use pill-testing facilities if they were available; and (ii) they would not use the pills if testing (whether on-site or laboratory-based) showed them to be adulterated.

Agent-based modelling works by looking for convergence across combinations of variables and repeated testing of combinations [34]. After testing 36 combinations of market penetration and pill-testing levels, and repeating each combination 10 times, repetitions displayed strong convergence. The results (Fig. 1) show that if the market penetration of the adulterated ecstasy pills is $10 \%$, just over $30 \%$ of young psychostimulant users would need access to pill-testing in order to ensure that the prevalence of major medical condition remained at less than 5\% (Fig. 1: scenario 1). If the market penetration of the adulterated pills increased to $20 \%$, approxi- mately $65 \%$ of young psychostimulant users would need access to pill-testing to ensure that the prevalence of major medical conditions remained at less than 5\% (Fig. 1: scenario 2). Finally, if the market penetration of the adulterated pills increased to $50 \%$, approximately $85 \%$ of young psychostimulant users would need access to pill-testing to ensure that the prevalence of major medical conditions remained at less than 5\% (Fig. 1: scenario 3). This simulation suggests that in situations where adulterated ecstasy pills become widely available in a drug market, pill-testing may be a potentially effective means of reducing the acute harms associated with their use.

\section{THREE CHALLENGES OF AGENT-BASED MODELLING}

Using agent-based modelling in our ethnoepidemiological work challenged the team in several ways. First, we had to learn how to manage a project that was not only multi-site and multi-method, but also multiepistemological. Over time, ongoing dialogue created a mutual respect between ethnographers, epidemiologists and modellers. This involved conducting each component in a disciplinary specific way but adopting the epistemological assumptions of other components when contributing to their development. For example, the requirement to simplify in order to further the agent-based modelling challenged the ethnographic preference for nuance and complexity. Similarly, the modellers had to modify their initial views of agents' behaviour based on utility func-
Figure I SimAmph ecstasy pill-testing scenarios

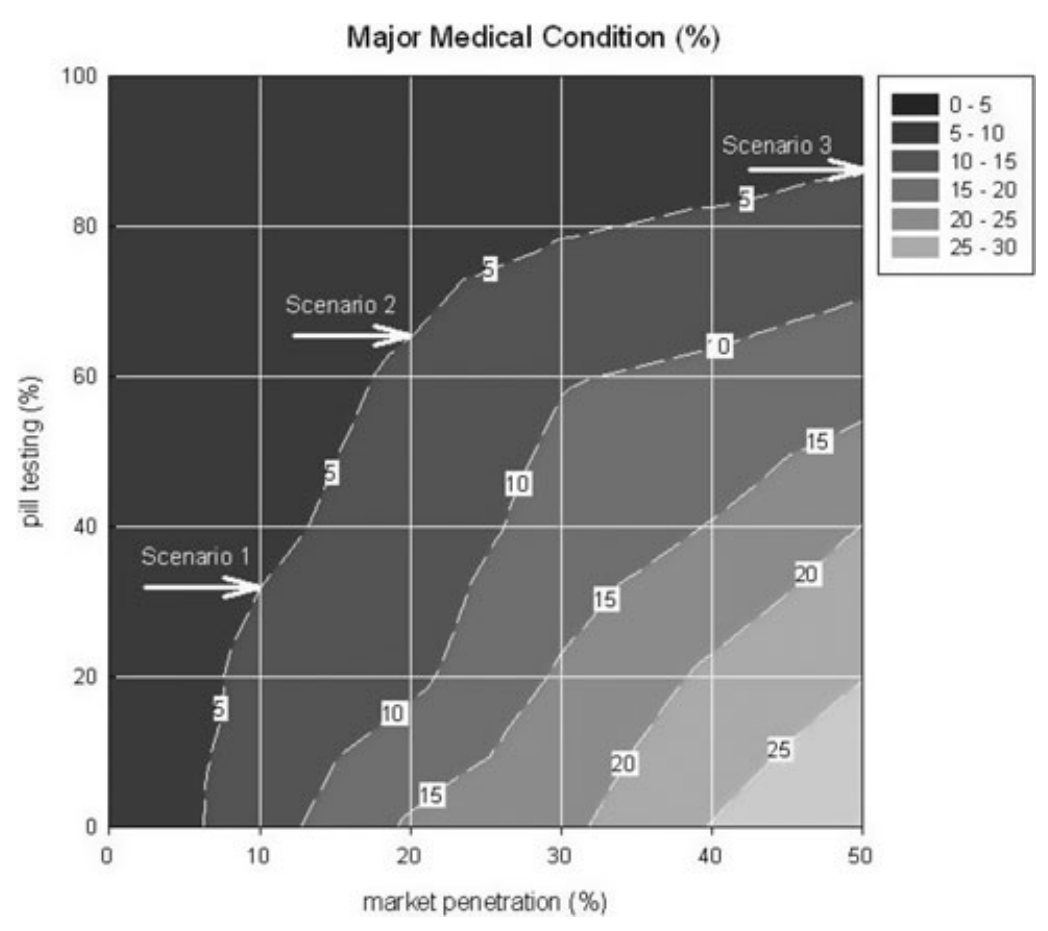


tions and develop agent dynamics based on social processes. They also had to alter their collective design methodology from a constructivist approach (where research team members create the model) to a more productive prototyping approach (where research team members provide feedback on a proposed model).

Secondly, although team discussions remained respectful, tensions did emerge in several areas. For example, doubts about our ability to integrate data on injecting and non-injecting drug use-in both the modelling and epidemiological work-were expressed in the first team meeting and throughout the project. Other team members felt it worth persevering with this ambitious form of integration but abandoned this aim when it became clear that the available project resources were insufficient. Agent-based models also work best when the data being used to create them can be quantified. In our case, this meant that several potentially important aspects of social context-for example, the impact on drug use of changes in sexual relationships-could not be included in SimAmph because our data did not allow us to assign them numerical values.

Thirdly, because team members had differing levels of exposure to agent-based modelling, we had to devote considerable time in team meetings to discussion of its rationale, principles and strengths and weaknesses. For example, basing some of SimAmph's behavioural parameters and assumptions on ethnographic data challenged team members more familiar with traditional mathematical modelling, which generally uses only epidemiological data. Another recurring theme in team discussions was the nature of the modelled outputs and how these should be best disseminated. While the model is well suited to testing plausible policy-relevant scenarios, it should be seen not as a predictive tool but as an aid to potential policy decisions. The research team was concerned about the risks of presenting the outcomes of modelled scenarios to policy makers, who might be less inclined to acknowledge the limitations of the model and any associated conclusions.

\section{CONCLUSION}

Agent-based modelling can be used to enhance the integration of ethno-epidemiological data on drug use. In our project it became a 'tool for managing the conversation', a way of structuring dialogue between the ethnographic and epidemiological components. Agent-based models can also be used to test various plausible scenarios, such as the probable impact of specific interventions on the prevalence of harms - a potentially important tool for policy and practice development. Such modelling goes beyond mathematical approaches to modelling of drug use and related harms by using ethnographic as well as epidemiological data to develop a more robust and ecologically valid model-that is, one that accords more closely with understandings of the social contexts of drug use. Finally, our project established a framework that facilitated multi-disciplinary collaboration, respected multiple epistemologies and allowed for the synthesis of diverse data in order to generate new knowledge relevant to the reduction of drug-related harms.

\section{Conflict of interest}

None.

\section{Acknowledgements}

The research reported in this paper was funded by National Health and Medical Research Council (NHMRC) Project Grant 323212. The study received approval from three university human research ethics committees. The National Drug Research Institute, National Centre for Epidemiology and Population Health, and National Centre in HIV Epidemiology and Clinical Research receive core funding from the Australian Government Department of Health and Ageing. Lisa Maher is supported by an NHMRC Senior Research Fellowship and Paul Dietze by an NHMRC Career Development Award. Caryn Anderson and Alison Ritter provided helpful comments on an earlier draft, as did two anonymous reviewers for Addiction. We also thank Paul McElwee for database development and support, and the research assistants who assisted with survey administration in Perth and Sydney. In particular, we are deeply indebted to the young psychostimulant users who participated in the research.

\section{References}

1. Rhodes T., Moore D. On the qualitative in drugs research: part one. Addiction Res Theory 2001; 9: 279-99.

2. Agar M. Recasting the 'ethno' in 'epidemiology. Med Anthropol 1996; 16: 391-403.

3. Bourgois P., Martinez A., Kral A., Edlin B. R., Schonberg J., Ciccarone D. Reinterpreting ethnic patterns among white and African American men who inject heroin: a social science of medicine approach. PLoS Med 2006; 3: e452.

4. Clatts M. C., Welle D. L., Goldsamt L. A., Lankenau S. E. An ethno-epidemiological model for the study of trends in illicit drug use: reflections on the 'emergence' of crack injection. Int J Drug Policy 2002; 13: 285-96.

5. Pach I. A., Gorman E. M. An ethno-epidemiological approach for the multi-site study of emerging drug abuse trends: the spread of methamphetamine in the United States of America. Bull Narcotic 2002; 54: 87-102.

6. Bryman A. Barriers to integrating quantitative and qualitative research. J Mixed Methods Res 2007; 1: 1-18.

7. Bammer G. Enhancing research collaboration: three key management challenges. Res Policy 2008; 37: 875-87.

8. Epstein J. M., Axtell R. Growing Artificial Societies: Social Science From the Bottom Up. Washington, DC: Brookings Institution Press; 1996. 
9. Liebrand W., Nowak A., Hegselmann R. Computer Modelling of Social Processes. London: Sage; 1988.

10. Weiss G. Multiagent Systems: A Modern Approach to Distributed Artificial Intelligence. Cambridge, MA: MIT Press; 1999.

11. Agar M. Agents in living color: towards emic agent-based models. J Artif Soc Soc Simulat 2005; 8: http://jasss. soc.surrey.ac.uk/8/1/4.html (last accessed 9 September 2009).

12. Agar M. H., Wilson D. Drugmart: heroin epidemics as complex adaptive systems. Complexity 2002; 7: 44-52.

13. Agar M. H. Another complex step: a model of heroin experimentation. Field Methods 2001; 134: 353-69.

14. Australian Institute of Health and Welfare (AIHW). 2004 National Drug Strategy Household Survey: Detailed Findings. Canberra: AIHW; 2005.

15. United Nations Office of Drugs and Crime. Ecstasy and Amphetamines: Global Survey 2003. New York: United Nations; 2003.

16. United Nations Office of Drugs and Crime (UNODC) Regional Center for East Asia and the Pacific. Patterns and Trends of Amphetamine-Type Stimulants (ATS) and Other Drugs of Abuse in East Asia and the Pacific 2006. Bangkok: UNODC; 2007.

17. Barker B., Degenhardt L., Breen C. Indicators of Drug Use, Harms and Treatment in Australia: Preliminary findings from the National Illicit Drug Indicators Project. Sydney: National Alcohol and Drug Research Council; 2003.

18. Kaye S., Darke S. A comparison of the harms associated with the injection of heroin and amphetamines. Drug Alcohol Depend 2000; 58: 189-95.

19. Topp L., Darke S. The applicability of the dependence syndrome to amphetamine. Drug Alcohol Depend 1997; 48: 113-5.

20. Wermuth L. Poverty and methamphetamine use: a study of AIDS risk behaviours among rural Northern Californian women. J Poverty 1999; 3: 25-45.

21. Australian National Council on Drugs (ANCD). Methamphetamines: Position Paper. Canberra: ANCD; 2007.

22. Drugs and Crime Prevention Committee. Inquiry into Amphetamine and 'Party Drug' Use in Victoria. Melbourne: Parliament of Victoria; 2004.

23. Dietze P. The relationship of ethnography to illicit drug surveillance. Int J Drug Policy 2003; 14: 131-5.

24. Gourley M. A subcultural study of recreational ecstasy use. J Sociol 2004; 40: 59-73.

25. Slavin S. Crystal methamphetamine use among gay men in Sydney. Contemp Drug Problems 2004; 31: 425-65.

26. Green R., Moore D. 'Kiddie drugs' and controlled pleasure: recreational use of dexamphetamine in a social network of young Australians. Int J Drug Policy; in press; 2009.

27. Siokou C., Moore D. 'This is not a rave!': changes in the commercialised Melbourne rave/dance party scene. Youth Stud Aust 2008; 27: 50-7.
28. Topp L., Hudson S., Maher L. Mental health symptoms among street-based psychostimulant injectors in Sydney's Kings Cross. Subst Use Misuse; in press; 2009.

29. Dray A., Perez P., Jones N., Le Page C., D’Aquino P., White I. et al. The AtollGame experience: from knowledge engineering to a computer-assisted role playing game. J Artif Soc Soc Simulat 2006; 9: http://jasss.soc.surrey.ac.uk/9/1/6.html (last accessed 9 September 2009).

30. Ferber J. Multi-Agent Systems: An Introduction to Distributed Artificial Intelligence. New York: Addison-Wesley; 1999.

31. Kaplan G. A. What's wrong with social epidemiology, and how can we make it better? Epidemiol Rev 2004; 26: 12435.

32. Perez P., Batten D.Complex science for a complex world: an introduction. In: Perez P., Batten D., editors. Complex Science for A Complex World: Exploring Human Ecosystems with Agents. Canberra: ANU EPress; 2006, p. 3-19.

33. Perez P., Dray A., Ritter A., Dietze P., Moore T., Mazerolle L. SimDrug: a multi-agent system tackling the complexity of illicit drug markets in Australia. In: Perez P., Batten D., editors. Complex Science for a Complex World: Exploring Human Ecosystems with Agents. Canberra: ANU EPress; 2006, p. 193-223.

34. Townsley M., Johnson S. The need for systematic replication and tests of validity in simulation. In: Liu L., Eck J., editors. Artificial Crime Analysis Systems: Using Comp Simulations and Geographic Information Systems. London: IGI Global Publisher; 2008, p. 1-18.

35. Bousquet F., Bakam I., Proton H., Le Page C. CORMAS: common-pool resources and multi-agent systems. Lect Notes Artif Intelligence 1998; 1416: 826-37.

36. Batty M., Torrens P. M. Modelling and prediction in a complex world. Future 2005; 37: 745-66.

37. Midgley D., Marks R., Kunchamwar D. Building and assurance of agent-based models: an example and challenge to the field. J Business Res 2007; 60: 884-93.

38. Duff C., Johnston J., Moore D., Goren N. 'Dropping, Connecting, Playing and Partying': Exploring the Social and Cultural Contexts of Ecstasy and Related Drug Use in Victoria. Melbourne: Premier's Drug Prevention Council; 2007.

39. Van De Wijngaart G., Braam R., De Bruin D., Fris M., Maalste N. J. M., Verbraeck H. T. Ecstasy use at large-scale dance events in the Netherlands. J Drug Iss 1999; 29: 679702 .

40. Benschop A., Rabes M., Korf D. J. Pill Testing, Ecstasy and Prevention: A Scientific Evaluation in Three European Cities. Amsterdam: Rozenberg Publishers; 2002.

41. Johnston J., Barratt M. J., Fry C. L., Kinner S., Stoove M., Degenhardt L. et al. A survey of regular ecstasy users' knowledge and practices around determining pill content and purity: implications for policy and practice. Int J Drug Policy 2006; 17: 464-72. 